Dy(III)-Based Metal-Organic Framework as a Fluorescent Probe for Highly Selective Detection of Picric Acid in Aqueous Medium

Richa Rajak, ${ }^{a}$ Mohit Saraf, ${ }^{b}$ Sanjay K. Verma, ${ }^{a}$ Ravinder Kumar and Shaikh M. Mobin*a,b,c

${ }^{a}$ Discipline of Chemistry, ${ }^{b}$ Discipline of Metallurgy Engineering and Materials Science and 'Discipline of Biosciences and Biomedical Engineering, Indian Institute of Technology Indore, Simrol, Khandwa Road, Indore 453552, India

*E-mail: xray@iiti.ac.in

Tel: +91 7312438752 


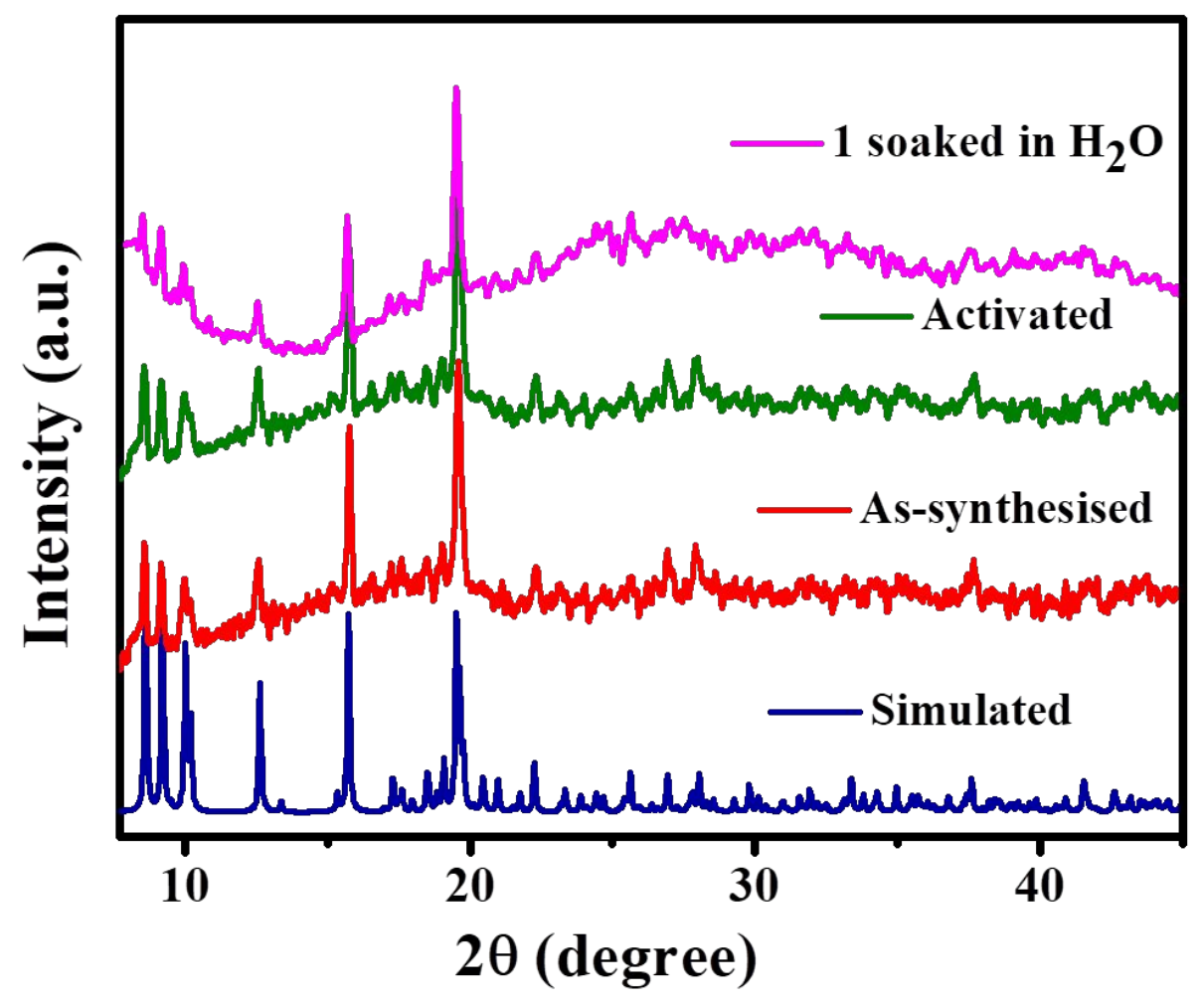

Figure S1. PXRD patterns of 1. 


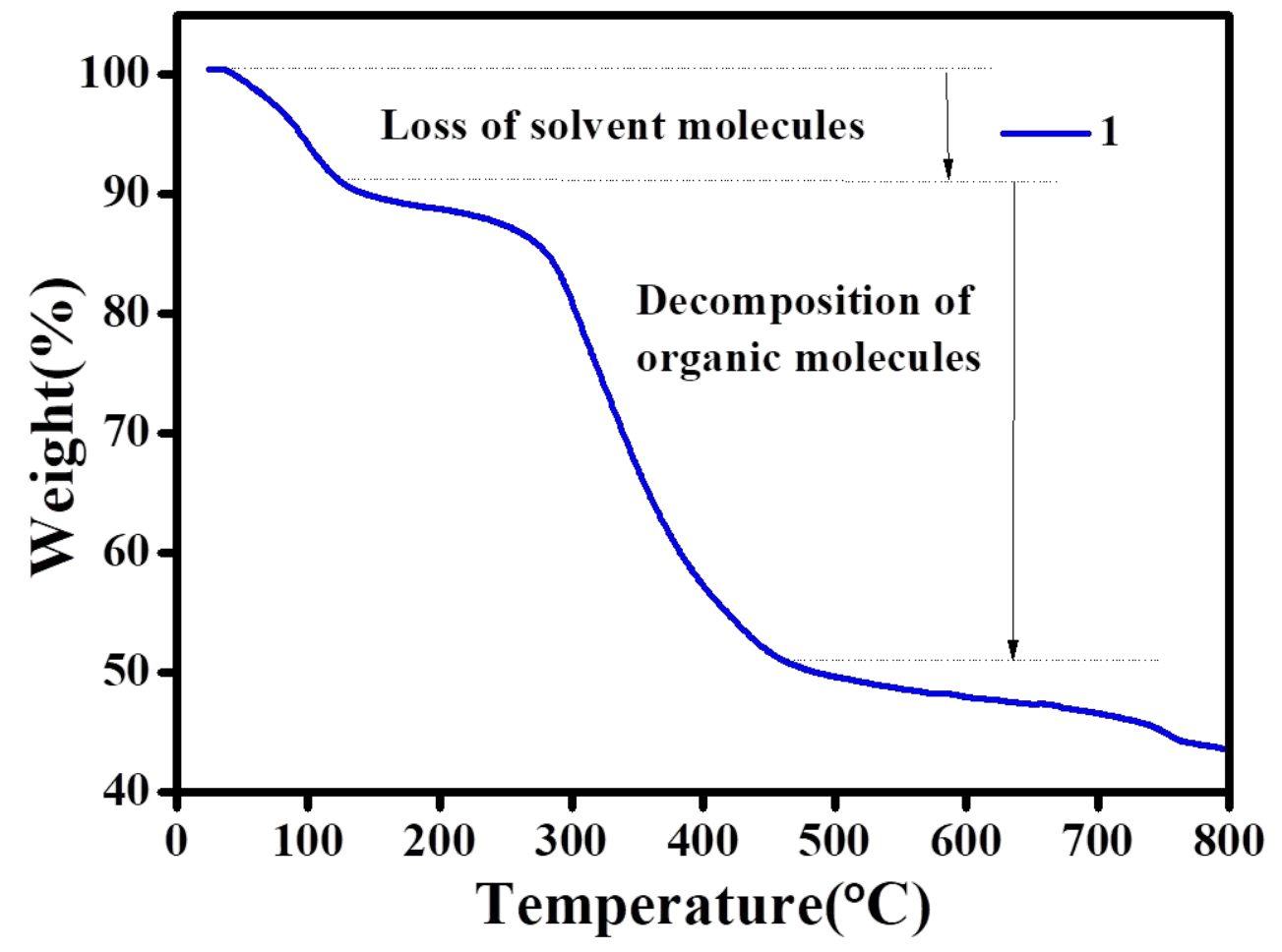

Figure S2. TGA profile of 1. 


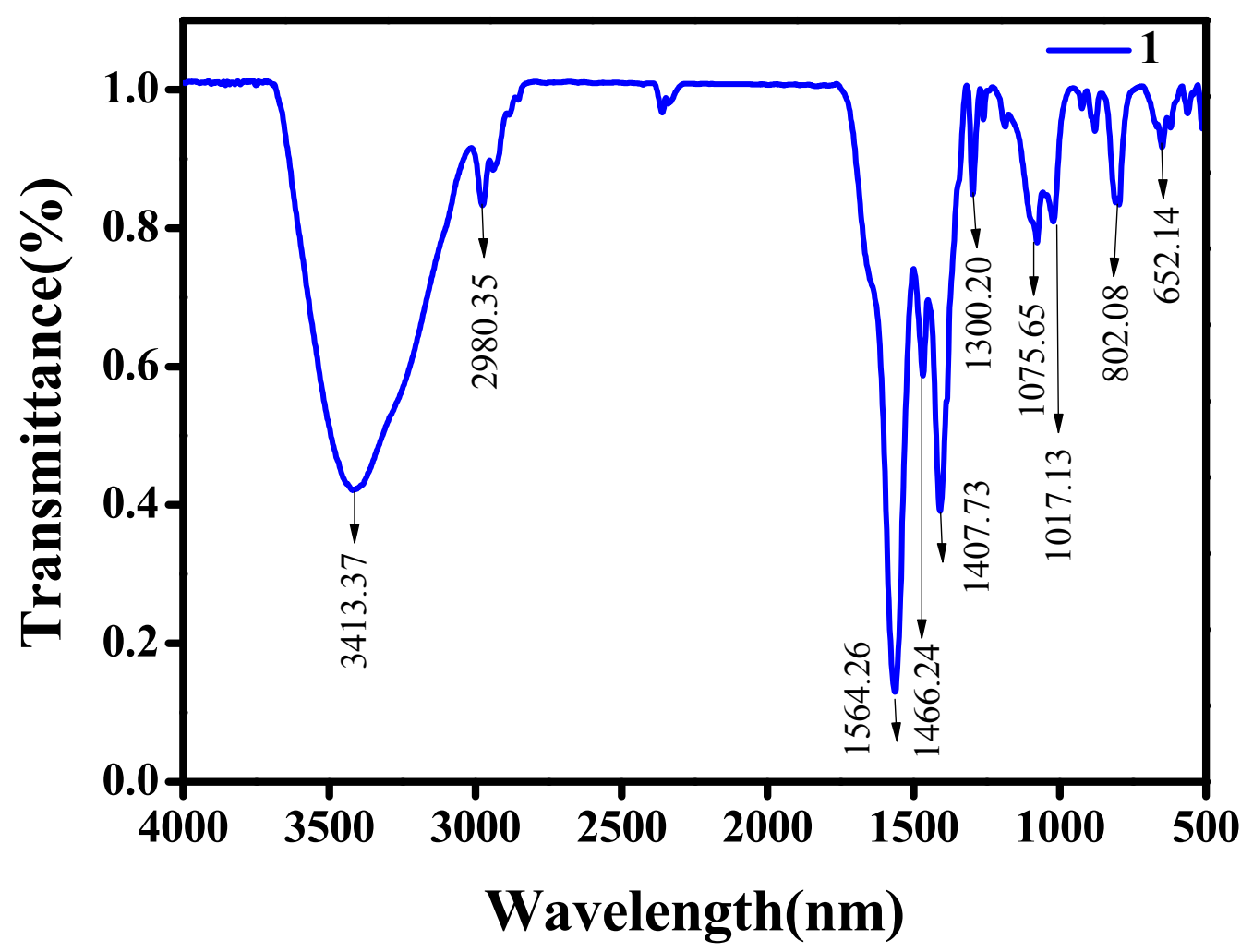

Figure S3. FT-IR of 1. 


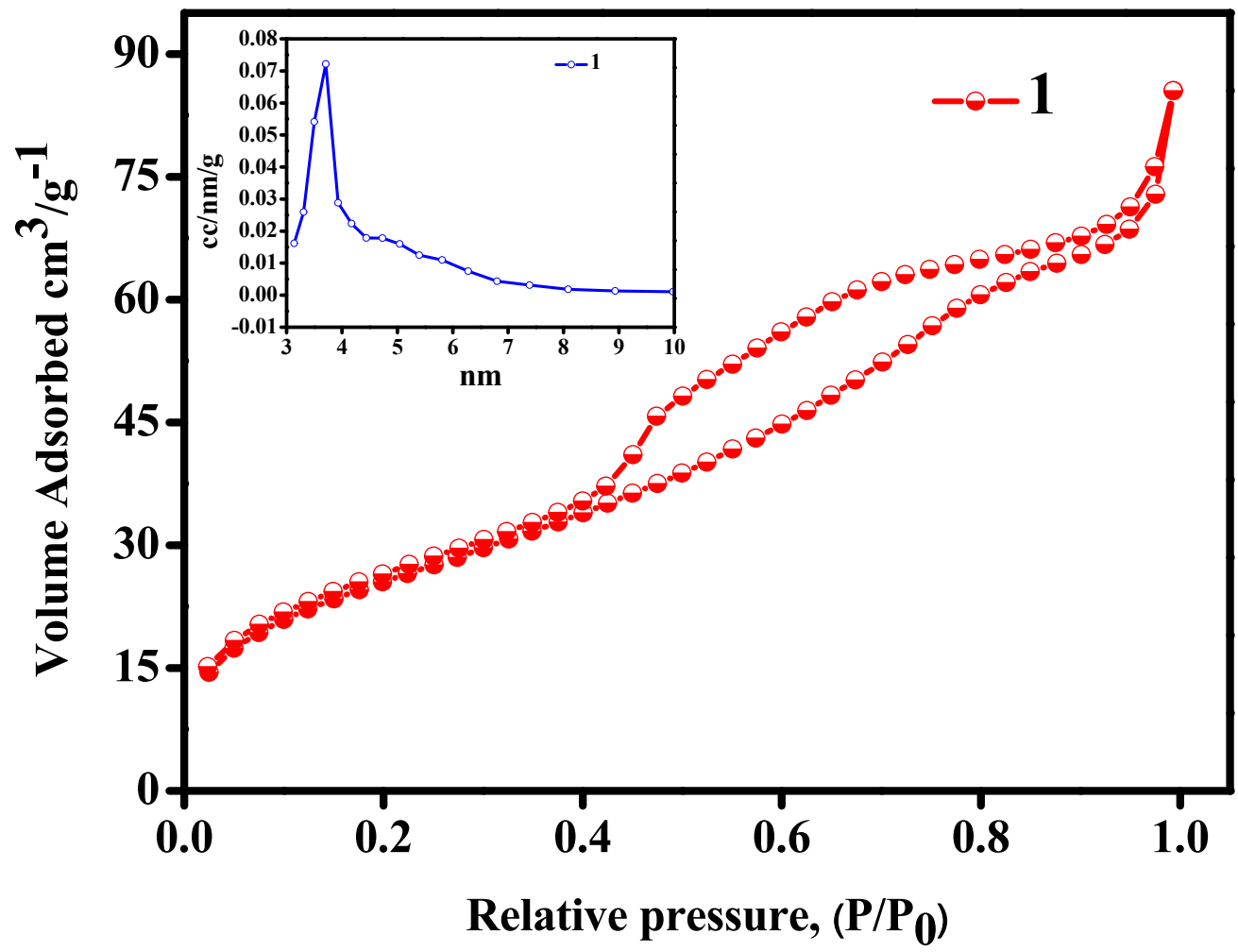

Figure S4. $\mathrm{N}_{2}$ isotherm and corresponsing BJH pore size distribution profile of $\mathbf{1}$. 


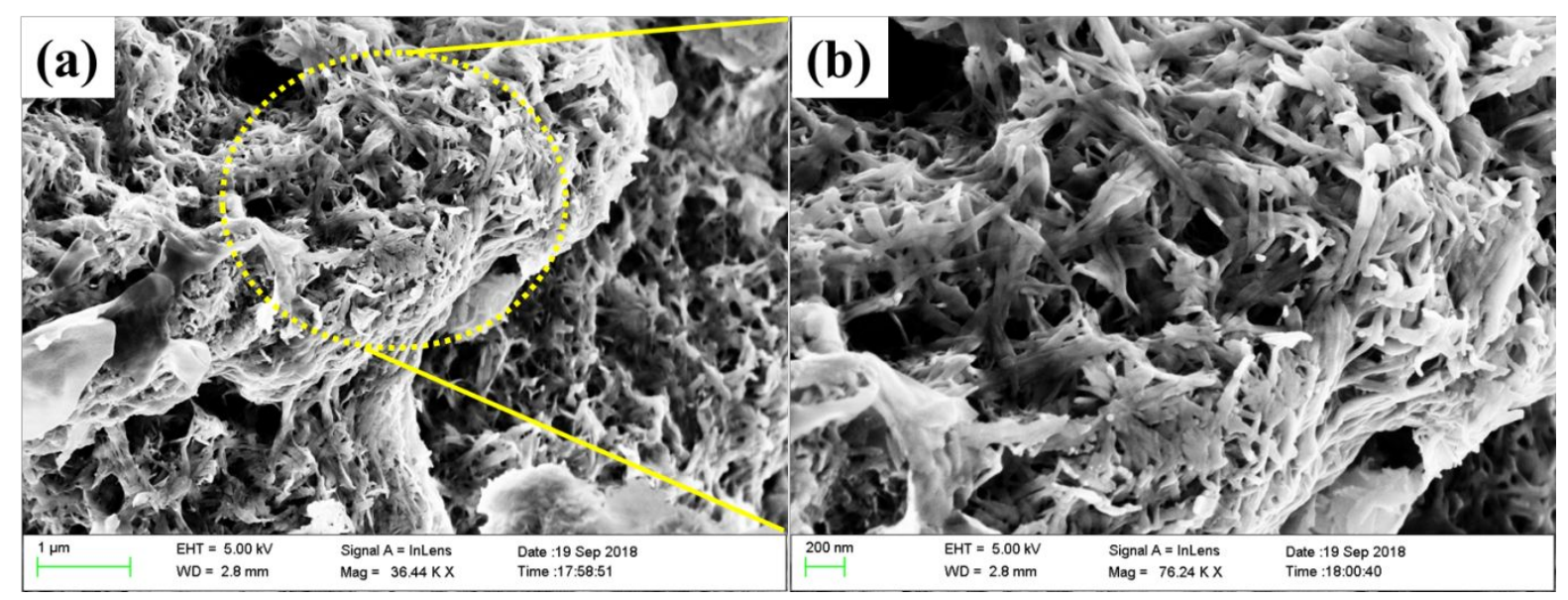

Figure S5. SEM images of $1(a, b)$. 


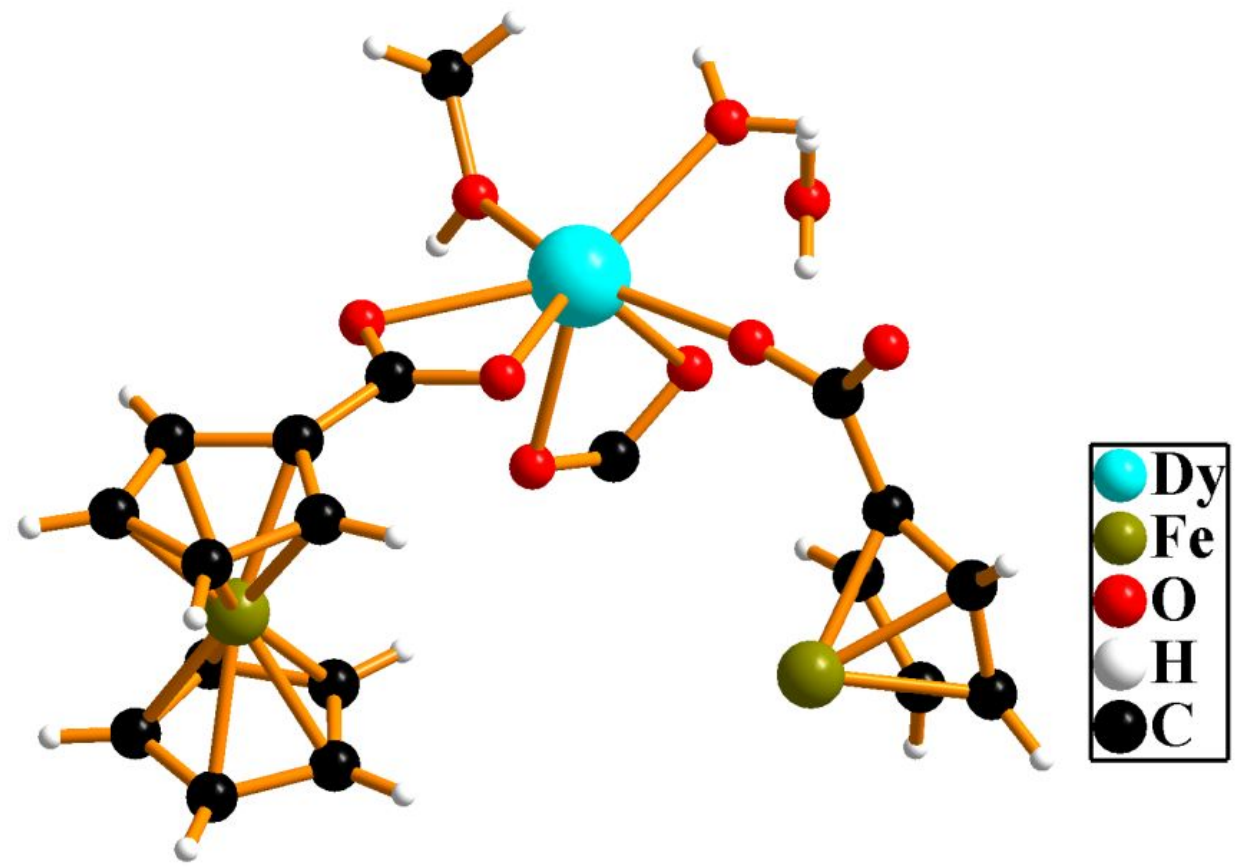

Figure S6. Assymetric unit of 1. Color Code: Dy (Cyan), Fe (Olive), O (Red), C (Black), H (white) 


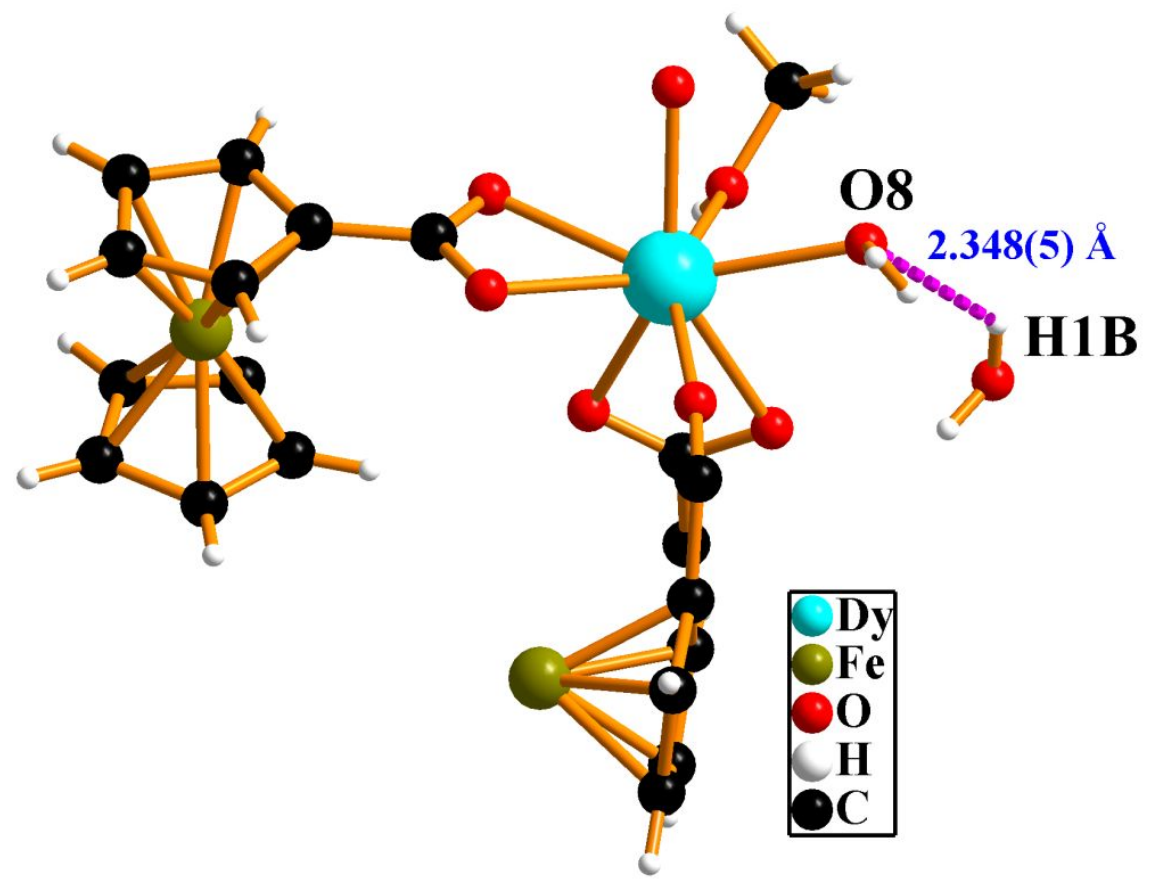

Figure S7. H-bonding interaction $\mathrm{O} 01-\mathrm{H} 1 \mathrm{~B} \cdots \mathrm{O} \mathrm{O} 8=2.348(5) \AA \AA$. Color Code: Dy (Cyan), Fe (Olive), O (Red), C (Black), H (white). 

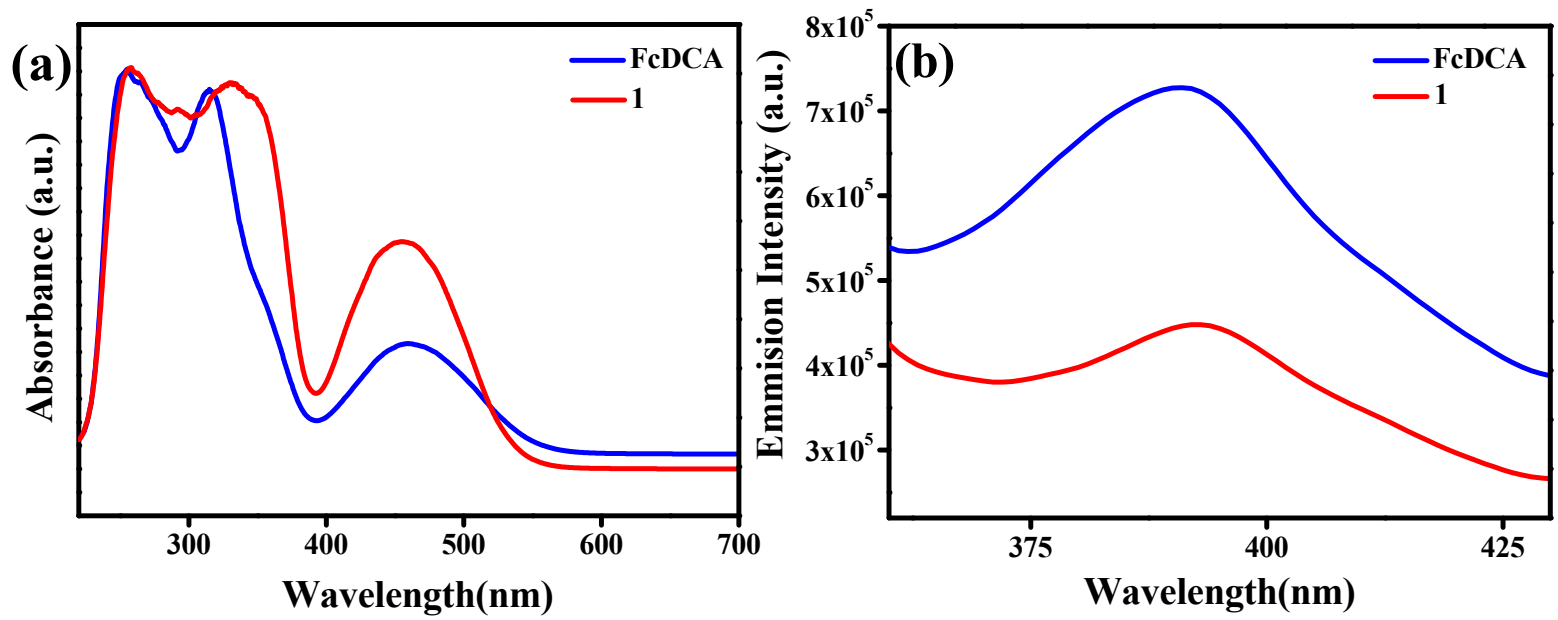

Figure S8. (a) Solid-state UV and (b) emission spectra of FcDCA and 1. 
The formula for calculating the fluorescence intensity quenching percentage of picric acid (PA):

$$
\left(I_{o}-I\right) / I_{\mathrm{o}} \times 100 \%
$$

Where, $I_{\mathrm{o}}=$ initial fluorescence intensity,

$I=$ intensity of 1 containing PA solution.
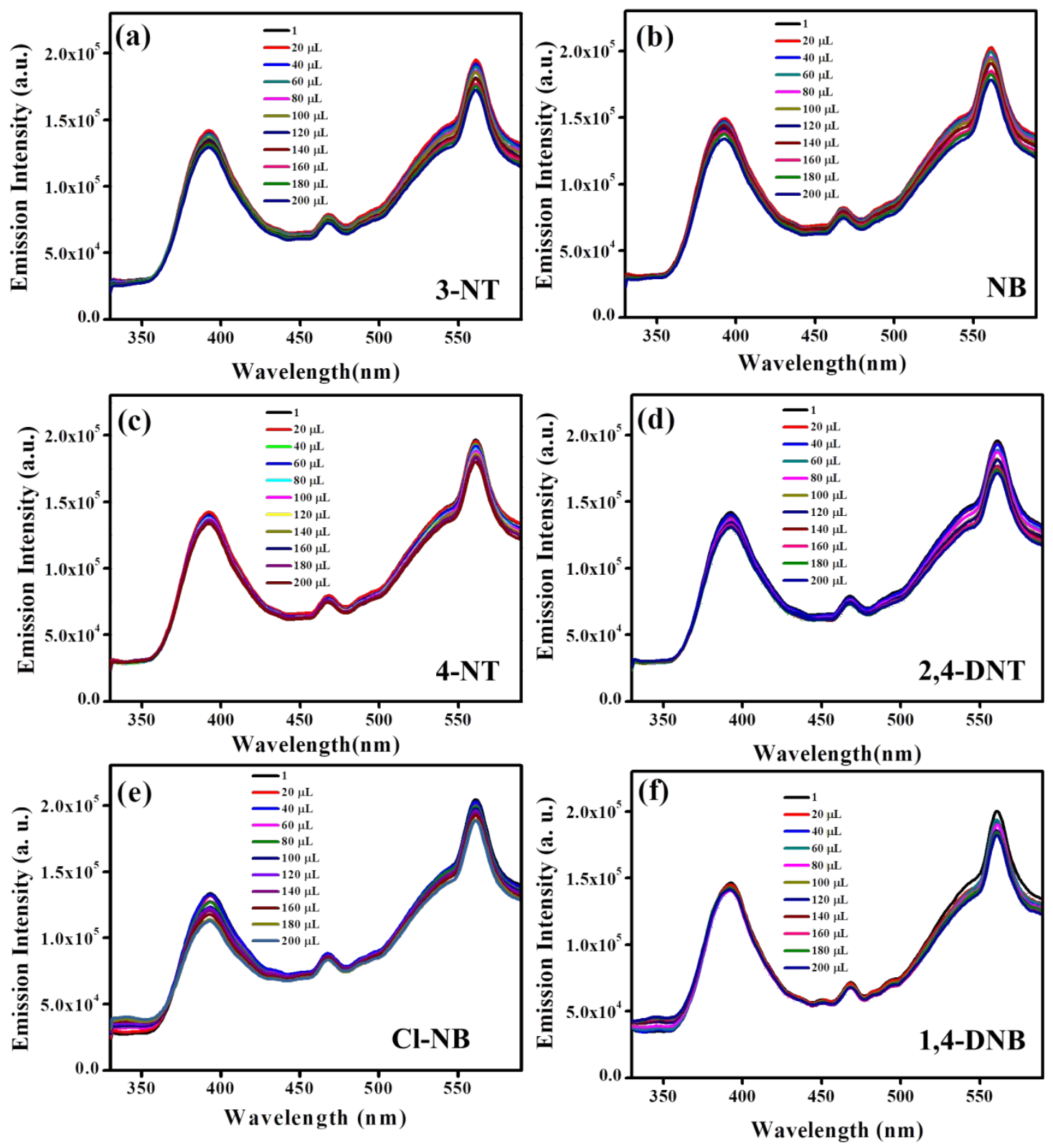

Figure S9. Emission spectra of 1 upon incremental addition of (a) 3-NT, (b) NB, (c) 4-NT, (d) 2,4-DNT, (e) Cl-NB, (f) 1,4-DNB (1mM) aqeous solution. 


\section{Stern-Volmer equation:}

$$
I_{0} / I=K_{\mathrm{Sv}}[\mathrm{A}]+1
$$

Where, $I_{0}=$ fluorescent intensity of $\mathbf{1}$ before the addition of the analyte $I=$ fluorescent intensity after the addition of the respective analyte

$$
K_{\mathrm{SV}}=\text { Stern-Volmer constant }
$$

$[\mathrm{A}]=$ molar concentration of the analyte $\left(\mathrm{M}^{-1}\right)$.

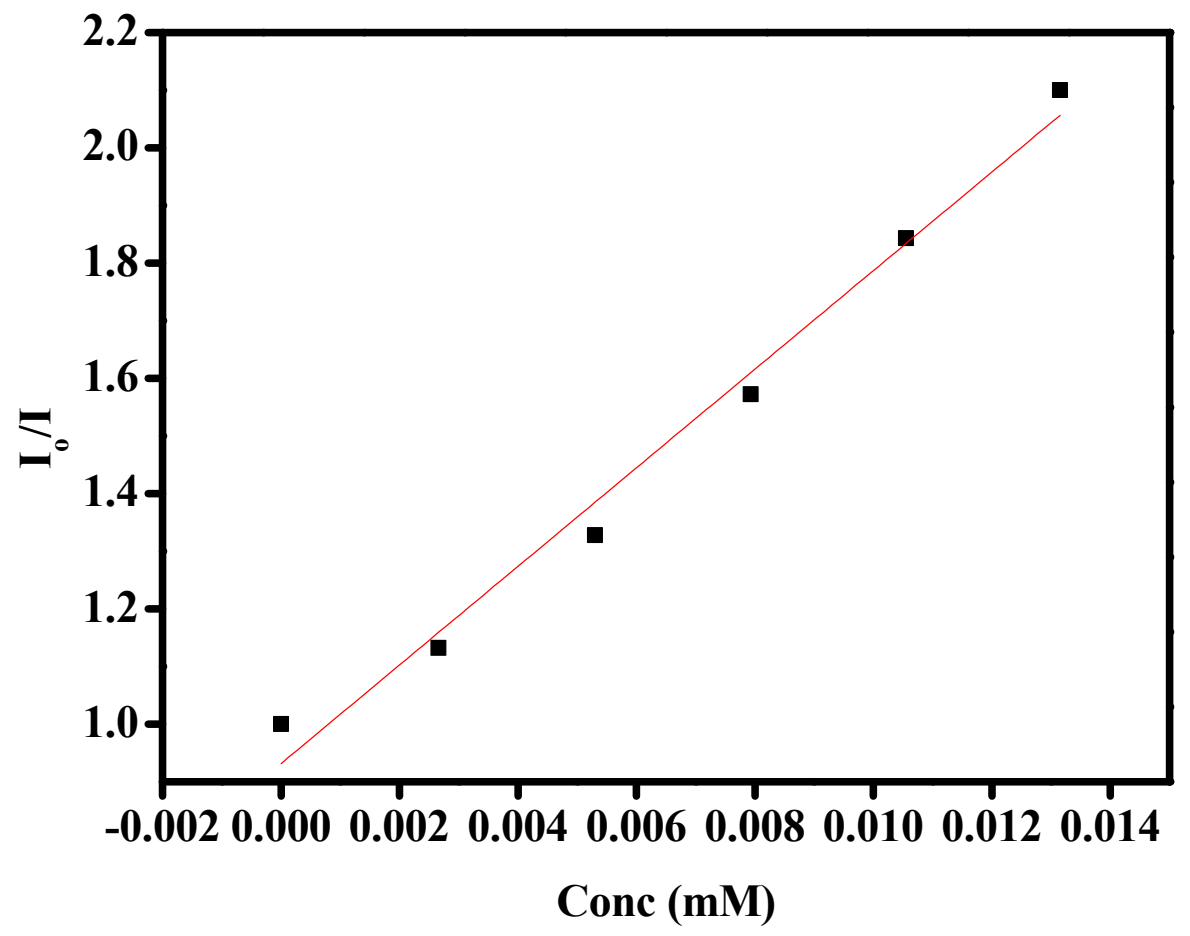

Figure S10. Fluorescence quenching of compound 1 by various concentration of PA, in which $\mathrm{I}_{0}$ and $\mathrm{I}$ denote the intensity of the fluorescence signal of the sensing material in the absence and presence of the analyte respectively. $\boldsymbol{I}_{\mathbf{0}} / \boldsymbol{I}=\boldsymbol{K}_{\mathrm{SV}}[\mathbf{P A}]+\mathbf{1}$ (Correlation coefficient, $\left.\mathrm{R}^{2}=0.983\right)$. 


\section{Calculation standard deviation and Detection limit}

Multiple black fluorescence spectra $(n=6)$ were recorded for the dispersed aqueous sample of Dy-MOF (1). Standard Deviation $\sigma$ for the blank probe without the addition of picric acid (PA) was calculated to be $\sigma=0.96$.

$$
\begin{aligned}
& \text { Detection Limit }=3 \sigma / \text { slope } \\
& =3 * 0.96 / 4.05 \\
& =0.71 \mu \mathrm{M}
\end{aligned}
$$

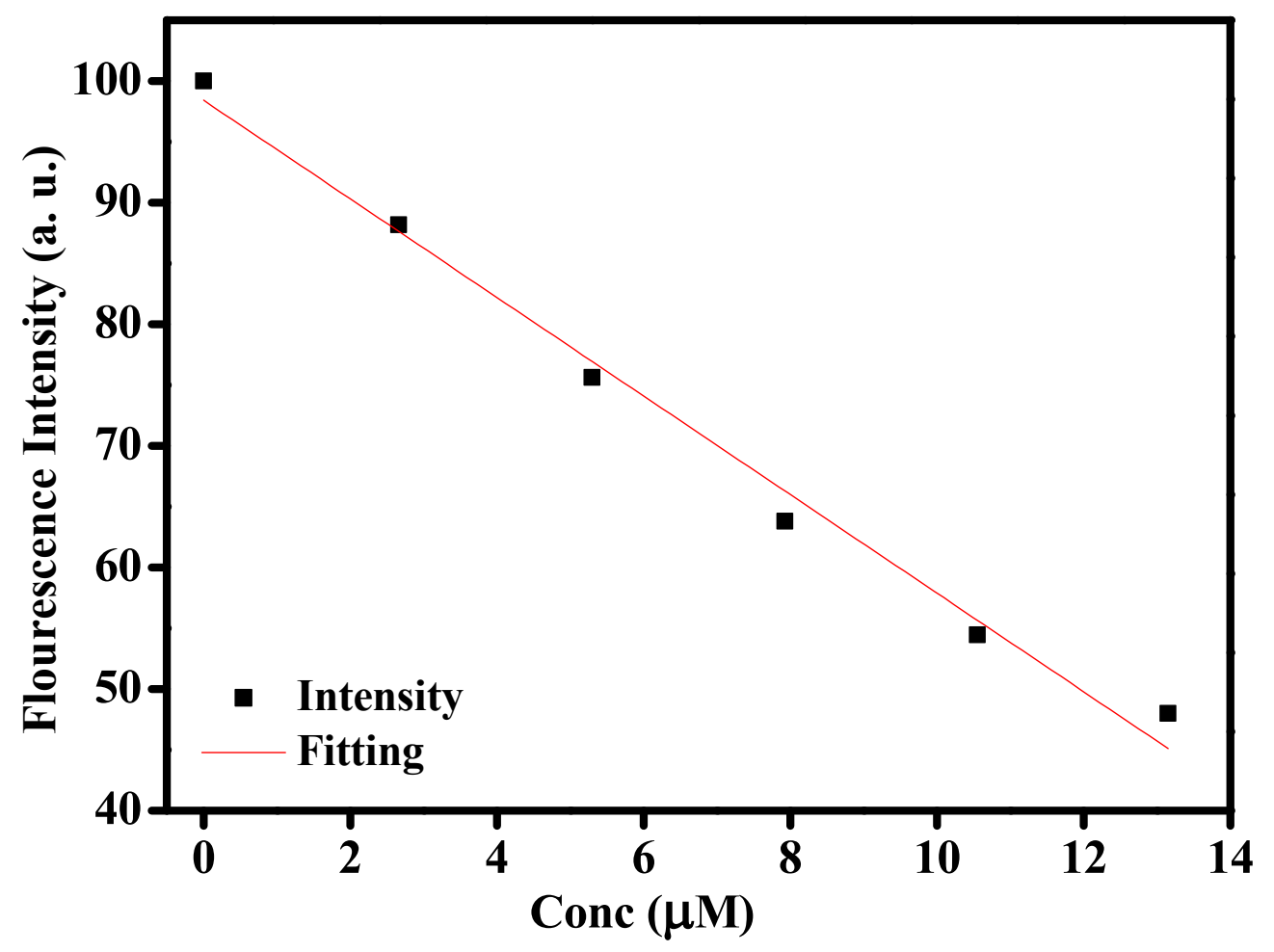

Figure S11. Relation of fluorescence intensity against PA added into the suspension of $\mathbf{1}$ and their linear fit curve for the estimation of $\operatorname{LOD}\left(\mathrm{R}^{2}=0.987\right)$. 


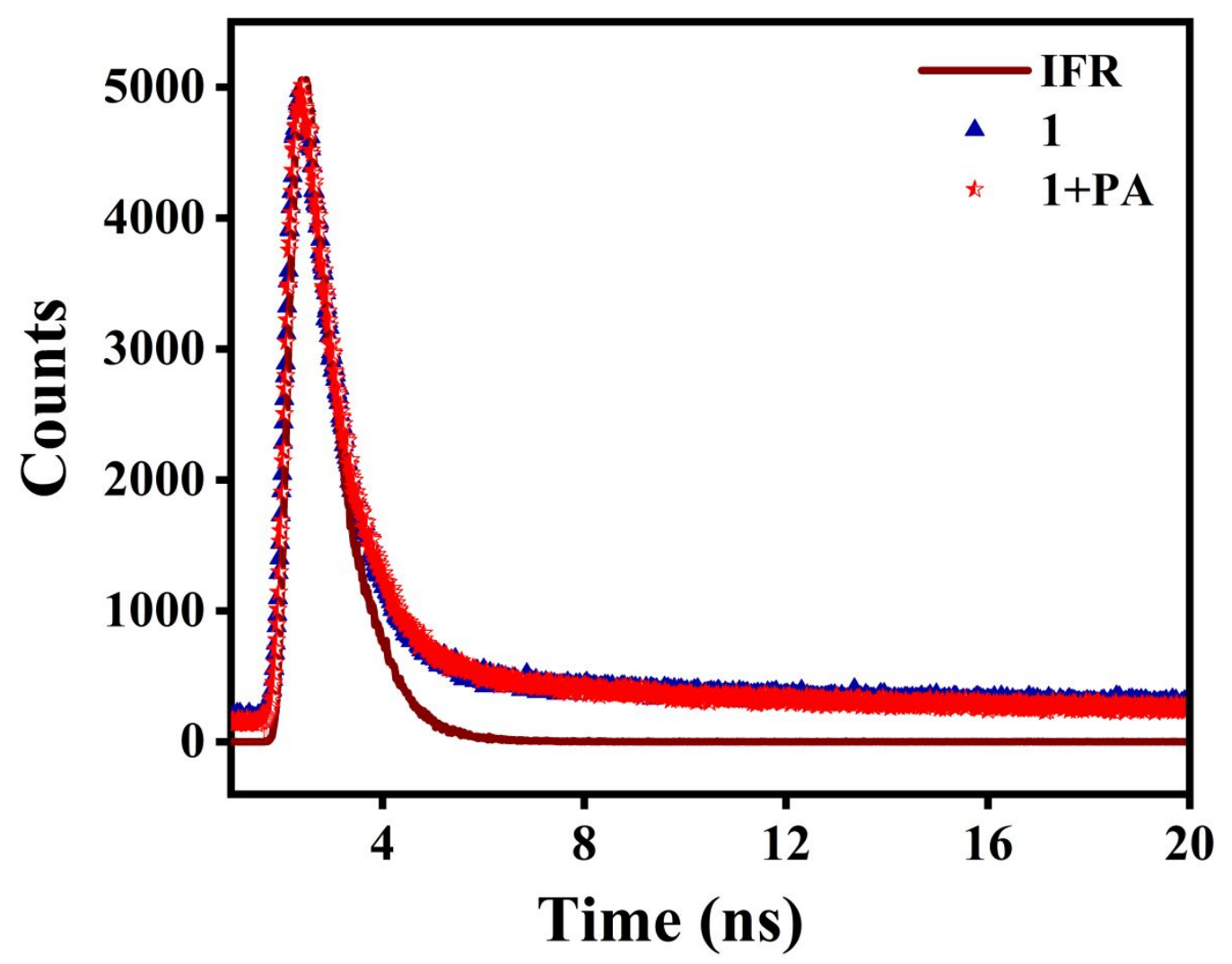

Figure S12. Fluorescence lifetime decay profile of $\mathbf{1}$ in the presence and absence of picric acid. $\left(\lambda \mathrm{ex}=393 \mathrm{~nm}\right.$, Life time of $\mathbf{1}=4.1 \times 10^{-11} \mathrm{~S}, \mathbf{1}+\mathbf{P A}=3.4 \times 10^{-11} \mathrm{~S}$ IRF $=$ Instrument Response Function). 

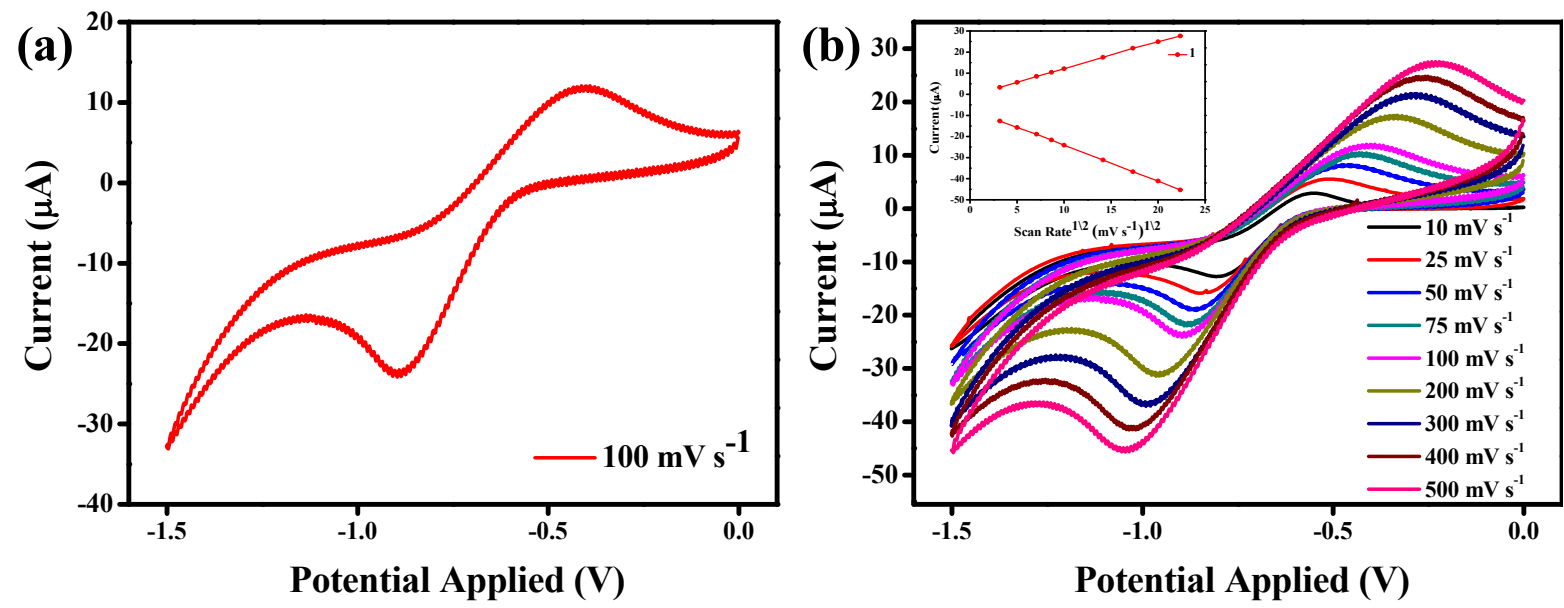

Figure S13. (a) CV profile of 1 at $100 \mathrm{mV} \mathrm{s}^{-1}$ in DMF, (b) CV profile of $\mathbf{1}$ at different scan rates (inset; Dependence of peak currents on scan rates). 
Table S1. Crystal data and structure refinement for $\mathbf{1 .}$

\begin{tabular}{|c|c|}
\hline Identification code & 1 \\
\hline empirical formula & $\mathrm{C}_{38} \mathrm{H}_{38} \mathrm{Dy}_{2} \mathrm{Fe}_{3} \mathrm{O}_{17}$ \\
\hline formula weight & 1259.23 \\
\hline crystal system & Monoclinic \\
\hline space group & $P 2 / n$ \\
\hline $\mathrm{a}(\AA)$ & $11.2595(6)$ \\
\hline $\mathrm{b}(\AA)$ & $10.2487(6)$ \\
\hline $\mathrm{c}(\AA)$ & $17.3417(10)$ \\
\hline$\alpha(\mathrm{deg})$ & 90 \\
\hline$\beta(\operatorname{deg})$ & $91.975(5)$ \\
\hline$\gamma(\mathrm{deg})$ & 90 \\
\hline $\mathrm{V}\left(\AA^{3}\right)$ & $2000.0(2)$ \\
\hline $\mathrm{Z}, \mathrm{d}_{\text {calcd }}\left(\mathrm{mg} \mathrm{m}^{-3}\right)$ & $2,2.091$ \\
\hline temperature $(\mathrm{K})$ & $298(2)$ \\
\hline Wavelength $(\AA)$ & 0.71073 \\
\hline$\theta$ range/ & 2.959 to 25.000 \\
\hline goodness-of-fit & 1.099 \\
\hline $\mathrm{R}_{1}^{\mathrm{a}}, \mathrm{wR}_{2}^{\mathrm{b}}[\mathrm{I}>2 \sigma(\mathrm{I})]$ & $\mathrm{R}_{1}=0.0317, \mathrm{wR}_{2}=0.0868$ \\
\hline $\mathrm{R}_{1}^{\mathrm{a}}, \mathrm{wR}_{2}^{\mathrm{b}}$ (all data) & $\mathrm{R}_{1}=0.0346, \mathrm{wR}_{2}=0.0892$ \\
\hline $\mathrm{F}(000)$ & 1224 \\
\hline Crystal size $\left(\mathrm{mm}^{3}\right)$ & $0.290 \times 0.240 \times 0.180$ \\
\hline$\mu / \mathrm{mm}^{-1}$ & 4.828 \\
\hline Index ranges & $-13 \leq h \leq 13,-12 \leq k \leq 12,-20 \leq l \leq 20$ \\
\hline Absorption correction & Semi-empirical from equivalents \\
\hline $\begin{array}{l}\text { Max. and min. } \\
\text { transmission }\end{array}$ & 1.00000 and 0.60521 \\
\hline Refinement method & Full-matrix least-squares on $\mathrm{F}^{2}$ \\
\hline Data/restraints/parameters & $3502 / 3 / 275$ \\
\hline $\begin{array}{l}\text { Largest diff. peak and hole } \\
\qquad\left(\mathrm{e} \AA^{-3}\right)\end{array}$ & 2.449 and -0.648 \\
\hline CCDC no. & 1917680 \\
\hline
\end{tabular}


Table S2. Bond lengths $[\AA]$ and angles $\left[{ }^{\circ}\right]$ for $\mathbf{1}$.

\begin{tabular}{|c|c|}
\hline \multicolumn{2}{|c|}{ Bond distances } \\
\hline $\mathrm{Dy}(1)-\mathrm{O}(2)$ & $2.293(4)$ \\
\hline $\mathrm{Dy}(1)-\mathrm{O}(1)$ & $2.316(5)$ \\
\hline $\mathrm{Dy}(1)-\mathrm{O}(3)$ & $2.380(4)$ \\
\hline $\mathrm{Dy}(1)-\mathrm{O}(7)$ & $2.386(4)$ \\
\hline $\mathrm{Dy}(1)-\mathrm{O}(6)$ & $2.404(3)$ \\
\hline $\mathrm{Dy}(1)-\mathrm{O}(8)$ & $2.420(4)$ \\
\hline Dy(1)-O(4) & $2.453(4)$ \\
\hline Dy(1)-O(5) & $2.485(4)$ \\
\hline \multicolumn{2}{|c|}{ Bond Angle } \\
\hline $\mathrm{O}(2)-\mathrm{Dy}(1)-\mathrm{O}(1)$ & $116.04(16)$ \\
\hline $\mathrm{O}(2)-\mathrm{Dy}(1)-\mathrm{O}(3)$ & $96.24(16)$ \\
\hline $\mathrm{O}(1)-\mathrm{Dy}(1)-\mathrm{O}(3)$ & $143.28(15)$ \\
\hline $\mathrm{O}(2)-\mathrm{Dy}(1)-\mathrm{O}(7)$ & $152.67(15)$ \\
\hline $\mathrm{O}(1)-\mathrm{Dy}(1)-\mathrm{O}(7)$ & $76.72(16)$ \\
\hline $\mathrm{O}(3)-\mathrm{Dy}(1)-\mathrm{O}(7)$ & $82.25(15)$ \\
\hline $\mathrm{O}(2)-\mathrm{Dy}(1)-\mathrm{O}(6)$ & $75.70(13)$ \\
\hline $\mathrm{O}(1)-\mathrm{Dy}(1)-\mathrm{O}(6)$ & $88.38(15)$ \\
\hline $\mathrm{O}(3)-\mathrm{Dy}(1)-\mathrm{O}(6)$ & $82.87(13)$ \\
\hline $\mathrm{O}(7)-\mathrm{Dy}(1)-\mathrm{O}(6)$ & $130.65(13)$ \\
\hline $\mathrm{O}(2)-\mathrm{Dy}(1)-\mathrm{O}(8)$ & $78.32(14)$ \\
\hline $\mathrm{O}(1)-\mathrm{Dy}(1)-\mathrm{O}(8)$ & $76.42(15)$ \\
\hline $\mathrm{O}(3)-\mathrm{Dy}(1)-\mathrm{O}(8)$ & $130.01(14)$ \\
\hline $\mathrm{O}(7)-\mathrm{Dy}(1)-\mathrm{O}(8)$ & $81.88(14)$ \\
\hline $\mathrm{O}(6)-\mathrm{Dy}(1)-\mathrm{O}(8)$ & $140.06(12)$ \\
\hline $\mathrm{O}(2)-\mathrm{Dy}(1)-\mathrm{O}(4)$ & $80.27(15)$ \\
\hline
\end{tabular}




\begin{tabular}{|c|c|}
\hline $\mathrm{O}(1)-\mathrm{Dy}(1)-\mathrm{O}(4)$ & $144.11(16)$ \\
\hline $\mathrm{O}(3)-\mathrm{Dy}(1)-\mathrm{O}(4)$ & $54.06(13)$ \\
\hline $\mathrm{O}(7)-\mathrm{Dy}(1)-\mathrm{O}(4)$ & $76.83(15)$ \\
\hline $\mathrm{O}(6)-\mathrm{Dy}(1)-\mathrm{O}(4)$ & $127.41(14)$ \\
\hline $\mathrm{O}(8)-\mathrm{Dy}(1)-\mathrm{O}(4)$ & $76.21(14)$ \\
\hline $\mathrm{O}(2)-\mathrm{Dy}(1)-\mathrm{O}(5)$ & $128.46(13)$ \\
\hline $\mathrm{O}(1)-\mathrm{Dy}(1)-\mathrm{O}(5)$ & $73.43(14)$ \\
\hline $\mathrm{O}(3)-\mathrm{Dy}(1)-\mathrm{O}(5)$ & $72.79(14)$ \\
\hline $\mathrm{O}(7)-\mathrm{Dy}(1)-\mathrm{O}(5)$ & $77.35(13)$ \\
\hline $\mathrm{O}(6)-\mathrm{Dy}(1)-\mathrm{O}(5)$ & $53.29(12)$ \\
\hline $\mathrm{O}(8)-\mathrm{Dy}(1)-\mathrm{O}(5)$ & $146.50(14)$ \\
\hline $\mathrm{O}(4)-\mathrm{Dy}(1)-\mathrm{O}(5)$ & $123.06(13)$ \\
\hline
\end{tabular}

Symmetry transformations used to generate equivalent atoms:

$$
\begin{aligned}
& \# 1-x+1,-y,-z+1 \quad \# 2-x+3 / 2, y,-z+3 / 2 \\
& \# 3-x+1,-y+1,-z+1 \quad \# 4-x+1 / 2, y,-z+3 / 2
\end{aligned}
$$

